\title{
Auffälliger Tastbefund durch Patienten - welche Sorgfalt schuldet der Gynäkologe?
}

In einem Klageverfahren vor dem Oberlandesgericht Koblenz hat der Sachverständige zu der Frage des Gerichts, welche Sorgfaltsanforderungen den Gynäkologen träfen, wenn eine Patientin einen selbst erhobenen auffälligen Tastbefund schildere, folgendes ausgeführt:

„Es kann ... durchaus so sein und es ist auch öfters so, dass eine Patientin kommt und glaubt, etwas Auffälliges in der Brust ertastet $z$ u haben, was sich für einen erfahrenen Facharzt für Gynäkologie als nicht pathologischer Tastbefund herausstellt ... Ich habe ... auch eine eigene Sprechstunde und dort passiert es mir regelmäßig, etwa ein- bis zweimal die Woche, dass Patientinnen kommen und glauben, etwas Auffälliges ertastet zu haben, was ich aber nicht als pathologischen Tastbefund einschätze."

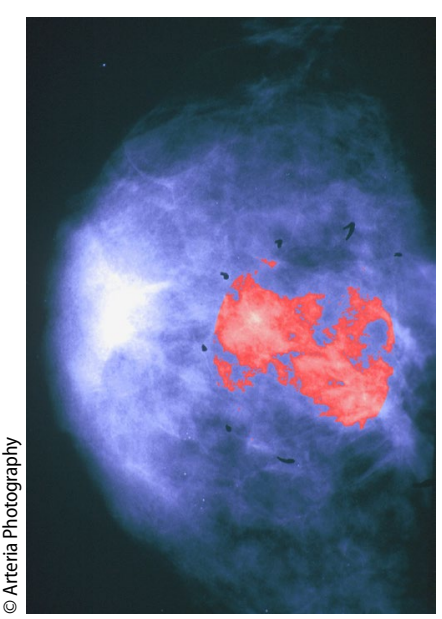

$\Delta$ Auch wenn die Patientin selbst eine Auffälligkeit ertastet, ist nicht unbedingt eine weitergehende Untersuchung geschuldet

Gynäkologe 2015 · 48:770-770 DOI 10.1007/s00129-015-3800-2 (c) Springer-Verlag Berlin Heidelberg 2015
Diese Äußerung des Sachverständigen bezog sich auf folgenden Sachverhalt:

Ein Facharzt für Frauenheilkunde und Geburtshilfe hatte bei einer Patientin, die er seit längerem betreute, die Verdachtsdiagnose eines rechtsseitigen Mammakarzinoms gestellt, die nachfolgend auch radiologisch und histologisch bestätigt wurde. Es wurde eine Ablatio erforderlich, der sich eine Chemotherapie und eine Strahlentherapie anschloss.

Die Patientin warf dem Facharzt für Frauenheilkunde und Geburtshilfe vor, er hätte weit früher zu seinem Befund gelangen müssen, so dass die Brust noch $\mathrm{zu}$ retten gewesen wäre. Sie habe ihm mehrfach von einem von ihr selbst erhobenen auffälligen Tastbefund berichtet. Der Facharzt für Frauenheilkunde und Geburtshilfe hätte regelmäßig die Brust untersucht, aber nichts Auffälliges feststellen können. Die Patientin warf dem Facharzt für Frauenheilkunde und Geburtshilfe vor, er habe sich nicht auf die palpatorische Befunderhebung beschränken dürfen, sondern eine Mammographie veranlassen müssen.

Das Landgericht Koblenz hat die Klage der Patientin auf Schadenersatz abgewiesen, weil es keinen Behandlungsfehler erkennen konnte, das Oberlandesgericht Koblenz hat sich dieser Entscheidung angeschlossen.

In den Gründen dieser Entscheidung hat das Oberlandesgericht Koblenz zunächst - gestützt auf die Ausführungen des Sachverständigen - bestätigt, dass die Palpation Grundlage des Vorsorgesystems ist und eine weitergehende Untersuchung - bei negati- vem Befund der Palpation - auch dann nicht geschuldet ist, wenn die Patientin von einem selbst erhobenen auffälligen Tastbefund berichtet. Dass der haftungsrechtliche Sorgfaltsmaßstab nicht weiterreichen kann als derjenige, der sozialrechtlich im Rahmen des Vertragsarztsystems ist eine zu begrüßende Grundaussage des Beschlusses des Oberlandesgerichts Koblenz vom 4.7.2014.

Allerdings liegt, wie zumeist, der Teufel im Detail, so dass derjenige, der meint, den Beschluss des Oberlandesgerichts Koblenz als generelle Richtschnur verstehen zu müssen, diesen fehlinterpretieren würde.

Die Patientin im Fall des Oberlandesgerichts Koblenz war bei dem Gynäkologen mehrfach mit auffälligem selbst erhobenem Tastbefund vorstellig geworden. Angesichts der interdisziplinären S3-Leitlinie für Diagnostik, Therapie und Nachsorge des Mammakarzinoms (allerdings bei auffälligem bzw. suspekten Befunden der Mamma) sind notwendige Basisuntersuchungen die klinische Brustuntersuchung (Inspektion, Palpation von Brust- und Lymphanschlussgebieten) Mammographie und Ultraschall. Dies lässt schon die Frage stellen, ob nicht bei einem mehrfach von der Patientin berichteten auffälligen selbst erhobenen Tastbefund eine Mammographie angezeigt gewesen wäre.

Zudem muss in der Praxis bedacht werden, dass die Entscheidung eines Gerichts anders ausfallen kann, wenn ein anderer Sachverständiger den Fall beurteilt oder aus den zeitlichen Abläufen sich konkrete Anhaltspunkte für eine möglicherweise unzureichende Palpation ergeben. Hätte nicht der Umstand, dass die Patientin im Februar bei dem Gynäkologen letztmalig mit vermeintlich auffälligem Tastbefund vorstellig geworden ist in Verbindung damit, dass Ende Juni die Verdachtsdiagnose des Mammakarzinoms bestätigt wurde, zu der Frage führen müssen, ob der Tumor nicht bereits im Februar tastbar gewesen wäre?

Der Beschluss des Oberlandesgerichts Koblenz vom 4.7.2014 eignet sich also als generelle Leitlinie für die Praxis nur begrenzt.

\section{Korrespondenzadresse}

RA Dr. Horst Bonvie

Sieker Landstr. 3

22927 Großhansdorf

bonvie@bonvie-medizinrecht.de

Zusammenfassung aus Bonvie $\mathrm{H}$ (2015) MedR 33:351-353,

DOI: 10.1007/s00350-015-3995-1

\section{Infobox}

Weitere interessante Beiträge aus der Zeitschrift MedR Medizinrecht, Ausgabe 05/2015 finden Sie unter folgendem Link: http://link.springer.com/journal/ $350 / 32 / 7 /$ page/1

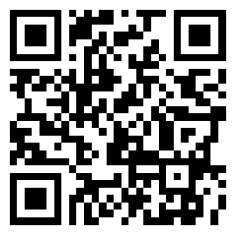

Oder gehen Sie mit Ihrem Smartphone direkt auf die Homepage der Zeitschrift MedR Medizinrecht. 